\title{
Non-monotonic piezoresistive behaviour of graphene nanoplatelet (GNP)-polymer composite flexible films prepared by solvent casting
}

\author{
S. Makireddi ${ }^{1}, F$. V. Varghes $^{2}$, K. Balasubramaniam ${ }^{3 *}$ \\ ${ }^{1}$ Aircraft Research and Design Centre, Hindustan Aeronautics Limited, 560037 Bengaluru, India \\ ${ }^{2}$ Defence Bioengineering and Electromedical Laboratory, 560037 Bengaluru, India \\ ${ }^{3}$ Center for Nondestructive Evaluation, Department of Mechanical Engineering, Indian Institute of Technology Madras, \\ 600036 Chennai, India
}

Received 14 December 2016; accepted in revised form 20 February 2017

\begin{abstract}
Graphene-polymer nanocomposite films show good piezoresistive behaviour and it is reported that the sensitivity increases either with the increased sheet resistance or decreased number density of the graphene fillers. A little is known about this behaviour near the percolation region. In this study, graphene nanoplatelet (GNP)/poly (methyl methacrylate) (PMMA) flexible films are fabricated via solution casting process at varying weight percent of GNP. Electrical and piezoresistive behaviour of these films is studied as a function of GNP concentration. Piezoresistive strain sensitivity of the films is measured by affixing the film to an aluminium specimen which is subjected to monotonic uniaxial tensile load. The change in resistance of the film with strain is monitored using a four probe. An electrical percolation threshold at 3 weight percent of GNP is observed. We report non-monotonic piezoresistive behaviour of these films as a function GNP concentration. We observe an increase in gauge factor (GF) with unstrained resistance of the films up to a critical resistance corresponding to percolation threshold. Beyond this limit the GF decreases with unstrained resistance.
\end{abstract}

Keywords: nanocomposites, graphene, percolation, piezoresistive behaviour, smart polymers

\section{Introduction}

Graphene and its derivatives, incorporated in a variety of polymer matrices as fillers are expected to find significant applications as smart materials and smart polymers in various fields like aerospace [1], flexible electronics [2], radiation protection [3], EMI/EMC [4] and for strain sensing in structural health monitoring (SHM) [5]. Graphene possess superior thermo-electro-mechanical properties with large specific surface area and higher aspect ratio. It demonstrates unusual electrical properties, such as anomalous quantum hall effect with high electron mobility of about $250000 \mathrm{~cm}^{2} /(\mathrm{V} \cdot \mathrm{s})$ at room temperature [6]. Electrical conductivity of graphene has been reported in the range of $10^{7}-10^{8} \mathrm{~S} / \mathrm{m}$. Tensile strength and Young's modulus of single layer graphene is about $130 \mathrm{GPa}$ and 1TPa respectively [7]. Owing to their outstanding properties, several derivatives of graphene such as graphite, expanded graphite (EG), foliated graphite (FG), graphene oxide (GO), reduced graphene oxide (RGO), functionalized graphene oxide (fGO) and graphene nanoplatelet (GNP) as fillers have been extensively studied to develop graphene/polymer nanocomposite films as piezoresistive strain sensors for structural health monitoring applications [8-11]. In situ polymerization, melt mixing and solution casting are some of the effective and commonly used methods to produce these nanocomposites. Among

$\overline{{ }^{*} \text { Corresponding author, e-mail: balas@iitm.ac.in }}$

(C) BME-PT 
these methods, solution casting is the most popular technique to fabricate polymer nanocomposites [12]. Polymers, such as polystyrene (PS), polycarbonate [13], polyacrylamide, polyimide [14] and poly (methyl methacrylate) (PMMA) [15] have been successfully used. Dispersion of graphene in the polymer matrix is still a challenge because of the strong tendency of the particles to aggregate [16]. Based on the degree of dispersion in the matrix, graphene fillers can exist in different forms e.g. stacked, intercalated or exfoliated. Exfoliated platelets have the largest interfacial contact with the polymer matrix owing to their high degree of dispersion [17] and hence shows enhanced electrical, mechanical and piezoresistive properties $[18,19]$.

Straining of the graphene nanocomposite film leads to a significant deformation of the graphene resulting in the shift of its electronic band structure. This cause changes in the electrical properties resulting in notable electro-elastic coupling and piezoresistivity [20]. Several groups have developed graphene nanocomposite strain sensors with different sensitivity, expressed as gauge factor (GF). Lee et al. [21] fabricated a graphene based strain sensor and reported a GF of 6.1 with an applied strain of $1 \%$. The 'transferred graphene' on polydimethylsiloxane (PDMS) has a higher GF of 151 [22]. A reduction in GF with increased unstrained resistance of the sensor and number density of graphene flakes was observed with GNP spray coated sensors [23]. Eswaraiah et al. [24] reported a GF of 12.1 at $2.2 \mathrm{wt} \%$ of RGO in a study with polyvinylidene fluoride (PVDF) matrix which in turn decreased with further loading of graphene. In yet another study with graphene wrapped over carbon nanotubes-based PVDF composites a GF of 20 was reported [25]. Increase in piezoresistive behaviour and GF was observed with increase in sheet resistance and a highest GF of 300 achieved at $10^{9} \Omega$ [26].

Although, highly sensitive sensors are reported using graphene nanocomposites, the effect of filler concentration, dispersion of nanofillers on the piezoresistive behaviour was not fully explored, particularly near the percolation region. At a certain filler loading known as percolation threshold, graphene fillers form a network leading to an abrupt rise in the electrical conductivity of the composite. Nanocomposites produced via melt mixing and solution mixing of graphene sheets have shown an electrical percolation threshold of 0.5 to $1 \%$ by volume in different polymer matrices [27, 28]. Liang et al. [29] reported a percolation threshold of 0.53 volume percent for the neat graphene/epoxy nanocomposites and 0.1 volume percent for the functionalized graphene filled epoxy composites. Pang et al. [30] reported percolation threshold as low as 0.07 volume percent for the graphene/ultrahigh molecular weight polyethylene nanocomposites. Numerous factors such as concentration and dispersion of graphene, fabrication method, functionalization and aspect ratio of graphene sheets, inter-sheet junction and orientation of graphene platelets influence the overall conductivity of the graphene/polymer composites and their percolation threshold and hence piezoresistivity [31, 32]. It is speculated that this critical weight percent and the state of dispersion is important for applications in the field of piezoresistive strain sensing [12, 20]. A detailed study with respect to percolation threshold and the piezoresistive behaviour of these graphene-polymer nanocomposites is essential and such understanding helps in the development of graphene based smart polymer nanocomposites and smart structures. We preferred solution casting process for the preparation of GNP/ PMMA films because the non-covalent interactions via hydrogen bonding and $\pi-\pi$ stacking are easily achieved by this method [33].

\section{Experimental}

\subsection{Materials and methods}

Pure and un-oxidized GNP of thickness $<3 \mathrm{~nm}$ and diameter $<10 \mu \mathrm{m}$ with an electrical conductivity of $70000 \mathrm{~S} / \mathrm{m}$, prepared by low temperature physical exfoliation were obtained from Graphenelab Ltd, London, UK and used without any further treatment. Carbobyk-9810 CNT paint, containing $8 \mathrm{wt} \%$ of CNT with water as a carrier was obtained from BYK Additives and Instruments, Wesel, Germany. Poly (methyl methacrylate) (PMMA) (average $M_{\mathrm{W}} \sim$ $120000 \mathrm{~g} / \mathrm{mol}$ ) purchased from Sigma-Aldrich, India and tetrahydrofuran (THF)-AR was used as the solvent, purchased from Ranbaxy Fine Chemicals, Mumbai, India. Conductive glue was obtained from Idolon Technologies, MA, US.

GNP was dispersed by adding $5 \mathrm{mg}$ of GNPs to $30 \mathrm{~mL}$ THF and ultrasonicated for 300 minutes using an ELMA Transonic Ti-H-5, $135 \mathrm{kHz}$ bath sonicator. PMMA/THF stock solution was separately prepared by dissolving $3.5 \mathrm{mg}$ of PMMA in $140 \mathrm{~mL}$ THF using a magnetic stirrer. GNP-THF dispersion was ultrasonicated for a further 180 minutes after adding $20 \mathrm{~mL}$ 


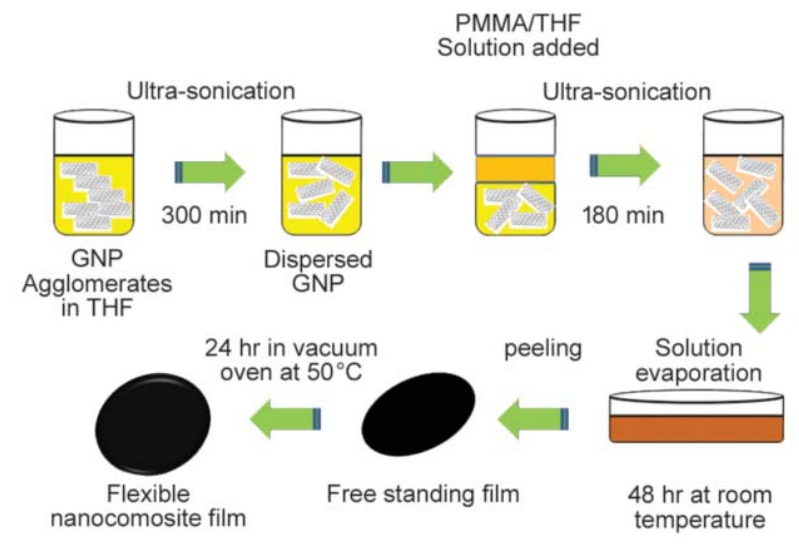

Figure 1. GNP/PMMA nanocomposite films preparation method

of stock solution. This mixture was poured in to a Petri dish and ultrasonicated further to avoid agglomeration of the GNP and the solvent was allowed to evaporate for $48 \mathrm{~h}$ at room temperature. The film was dried at $50^{\circ} \mathrm{C}$ for $24 \mathrm{~h}$ in a vacuum oven. This film is equivalent to $1 \mathrm{wt} \%$ GNP loading. The film thickness was measured to be approximately $100 \mu \mathrm{m}$. The steps involved in the preparation of the films are shown in Figure 1. Other weight percentage samples were prepared by varying the concentration of GNP in a similar manner.

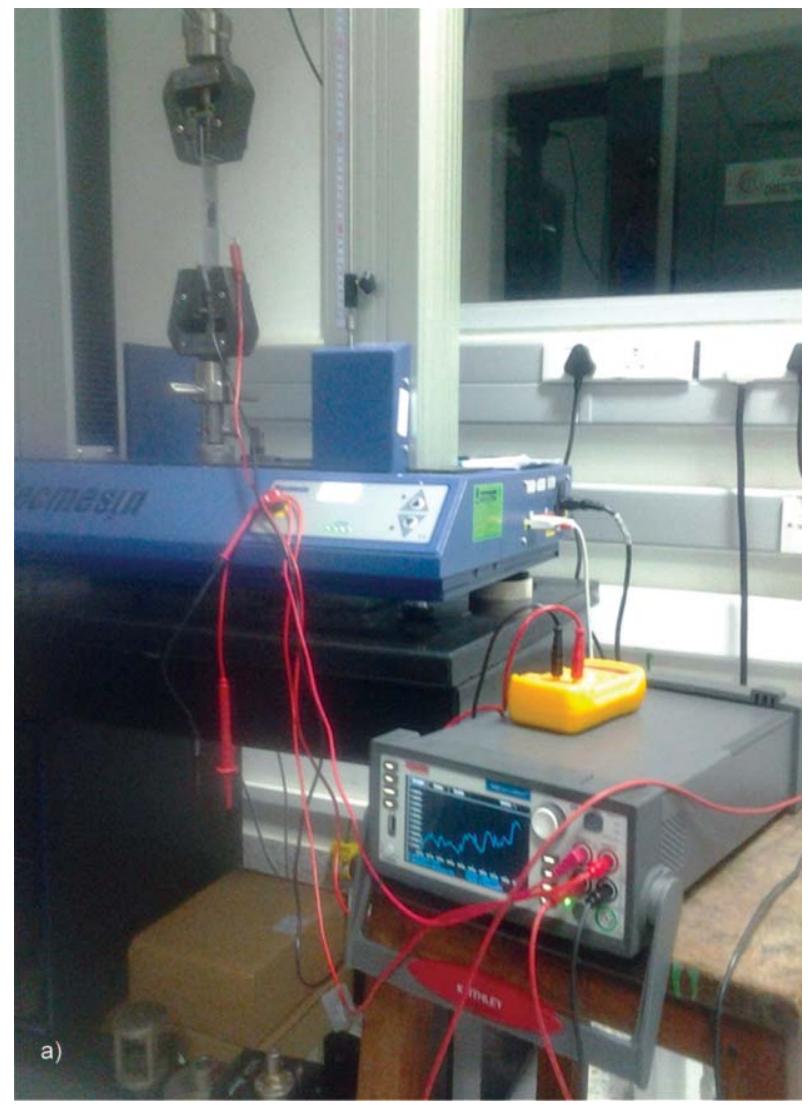

The electrical resistance of the nanocomposite films was measured on the surface using a Fluke 17B digital multimeter and expressed as bulk conductivity. To measure the strain sensitivity, films of $25 \times 15 \mathrm{~mm}$ size were affixed to aluminum beams of $200 \times 30 \times 2.5 \mathrm{~mm}$ dimensions by vacuum bonding using $\mathrm{M}$ bond 200 adhesive and cured for 24 hours. Measurement was done by subjecting the aluminum specimen to tensile load using a $10 \mathrm{kN}$ load cell (Figure 2a) and change in electrical resistance of the films was monitored using a four-probe Keithley 2450, Interactive Source Meter as shown in Figure 2b. The load and crosshead displacement were sampled at $1000 \mathrm{~Hz}$ frequency maintaining a loading rate of $1 \mathrm{~mm} / \mathrm{min}$. The measurements were repeated for all samples of varying GNP weight percentage. Similar type of measurements were conducted on the commercially obtained CNT paint coated on a polyethylene theraphthalate (PET) film and also on a conventional quarter bridge strain gauge WK-13-125TM-350 with a GF of $2.08 \pm 1 \%$.

\section{Results and discussion}

\subsection{Scanning electron microscopy (SEM)}

Scanning electron microscopy (SEM) of the nanocomposite samples were done to assess the dispersion

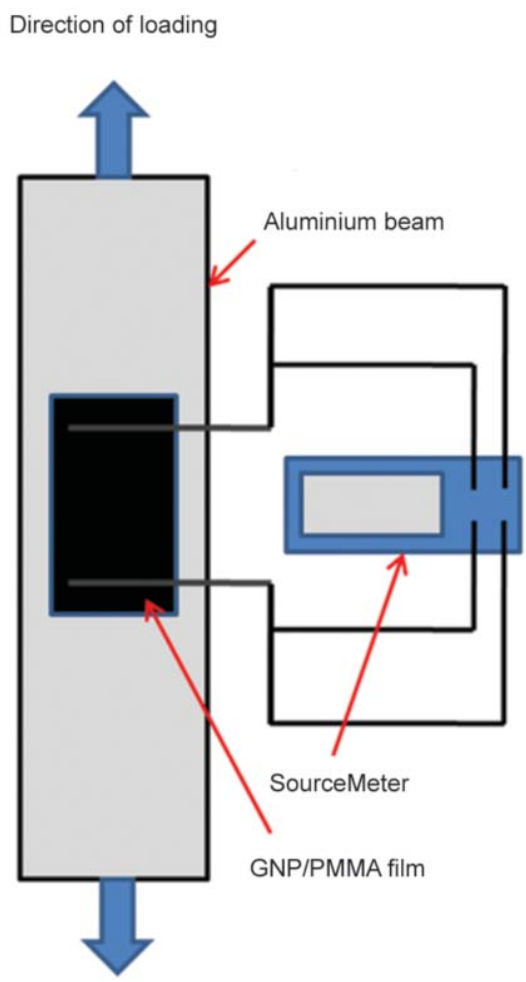

b)

Figure 2. a) Test setup to measure the strain sensitivity of GNP/PMMA films b) Schematic of the test setup (a film of size $25 \times 15 \mathrm{~mm}$ bonded to an aluminum beam of size $200 \times 30 \times 2.5 \mathrm{~mm}$ ) 


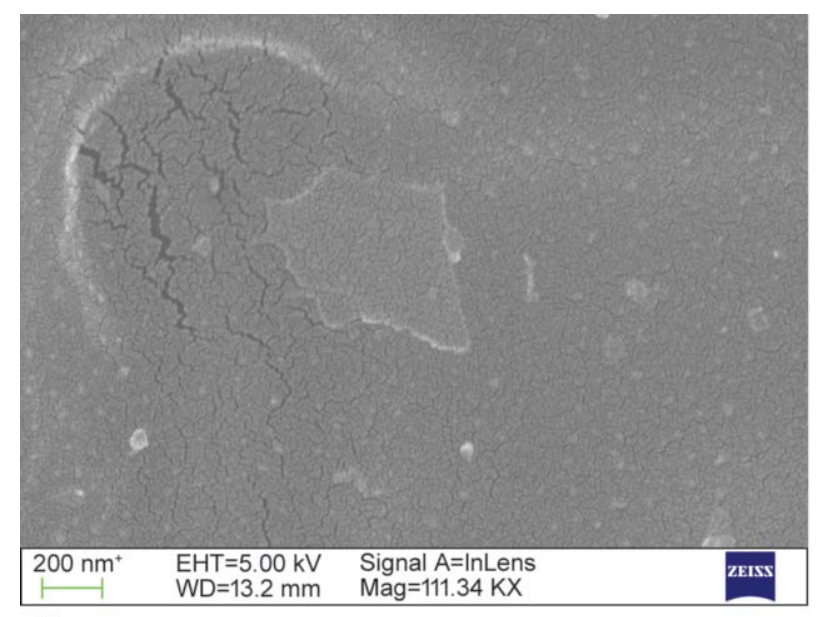

a)

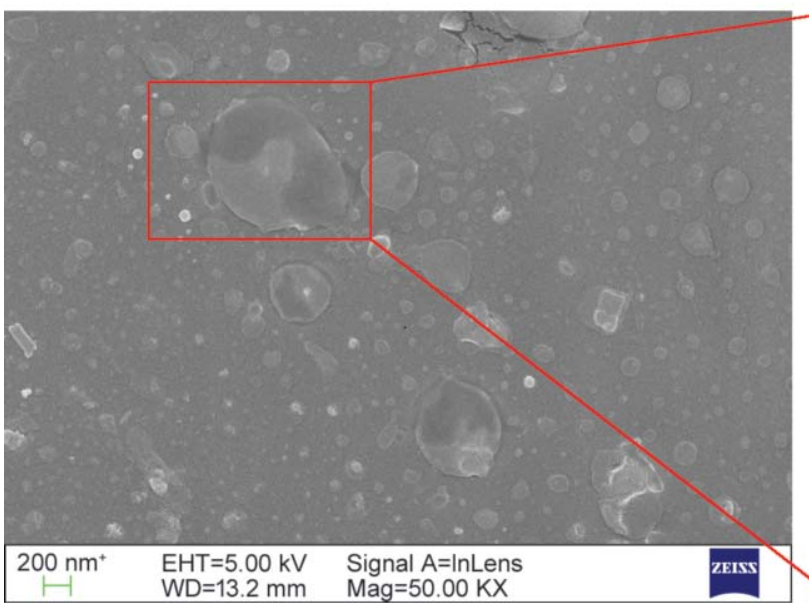

c)

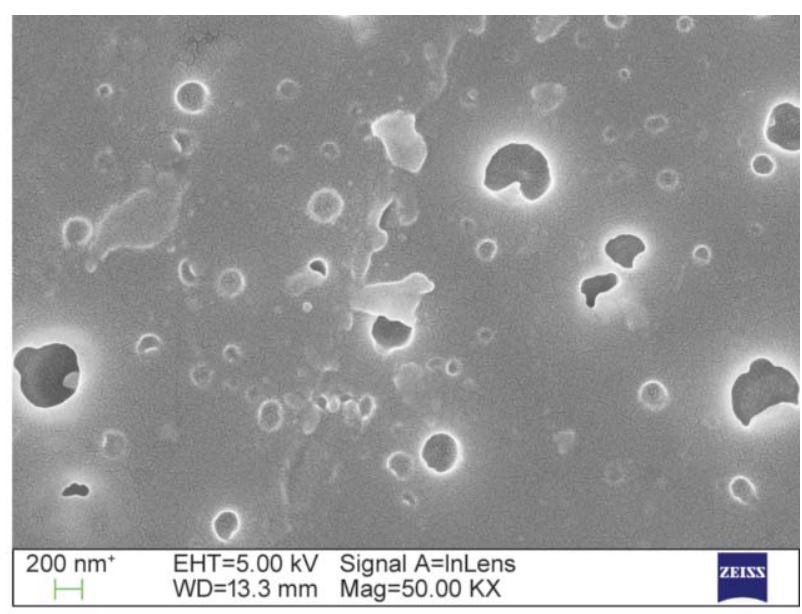

b)

Figure 3. SEM micrographs of GNP/PMMA nanocomposite films at (a) 2, (b) 3, (c) $10 \mathrm{wt} \%$ and (d) enlarged view of a the rectangular portion where agglomeration of several GNPs can be observed as a single graphene flake

of GNP in PMMA. Figures $3 a$ to $3 \mathrm{c}$ show the degree of dispersion at 2, 3 and $10 \mathrm{wt} \%$ GNP respectively. Platelet like flakes randomly distributed in the matrix can be identified. This distribution turns denser and the inter-particle distance of GNP becomes shorter as the weight percentage is increased. Homogeneous dispersion of nanofillers is a significant parameter for obtaining high performance nanocomposites. These GNPs observed to be oriented parallel to the film's surface in the SEM images. As reported earlier [14] nanoplatelet with high aspect ratio have a tendency to align and inclined to lay flat during the solution casting process. Figure $3 \mathrm{~d}$ shows the zoomed image of the particles where stacked flakes of size less than $2 \mu \mathrm{m}$ can be observed, indicating improper dispersion at higher filler loadings.

\subsection{Electrical conductivity}

Classic theory of percolation, envisages a power law relationship as shown in Equation (1) for conductive fillers incorporated in an insulating matrix: $\sigma_{\mathrm{c}}=\sigma_{0}\left(\phi-\phi_{\mathrm{c}}\right)^{\mathrm{t}}$

where $\sigma_{\mathrm{c}}$ is the conductivity of nanocomposite, $\sigma_{0}$ conductivity of the filler, $\phi$ filler fraction, $\phi_{\mathrm{c}}$ critical fraction of filler or percolation threshold, $t$ is critical exponent which is related to dimensional aspects of the filler network. According to this theory current can only flow above a critical fraction known as percolation threshold $\left(\phi_{\mathrm{c}}\right)$, hence the equation is valid for a region for $\phi>\phi_{\mathrm{c}}$. The bulk conductivity of the GNP/ PMMA films shows a power law dependency as a function of GNP weight percent (Figure 4). Initially, when the filler fraction is low the composite showed low conductivity in the orders of $10^{-3} \mathrm{~S} / \mathrm{m}$ which is a matrix dominated property. The bulk electrical conductivity of the film sharply increased to several orders of magnitude i.e. from 0.004 to $42.8 \mathrm{~S} / \mathrm{m}$ with subsequent increase in GNP concentration from 2 to $10 \mathrm{wt} \%$, arguably due to the building up of new conducting paths. In this range of GNP loading a certain critical weight percent probably exists where the inter 


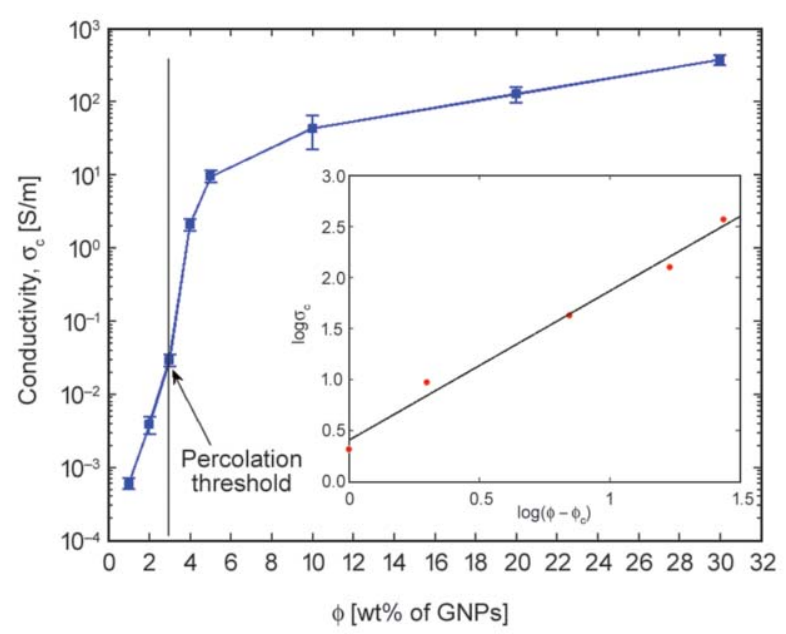

Figure 4. Electrical conductivity of the GNP/PMMA nanocomposite films as a function of GNP loading. Approximate inflexion point at $3 \mathrm{wt} \%$ was identified as percolation threshold $\phi_{\mathrm{c}}$. Data follows a power law and the data in the inset shows a linear trend for $\phi>\phi_{\mathrm{c}}$.

particle distance falls in the range required for tunneling of charge between the neighboring graphene flakes. This critical weight percent known as percolation threshold and the state of dispersion is important for applications in the field of piezoresistive strain sensing [12]. However from the Figure 4, we can observe that the increase in conductivity is really not as sharp as the percolation theory envisages. This can be attributed to the random and imperfect dispersion of the GNP in the matrix at higher concentrations. The exact point of percolation threshold is difficult to be located on this conductivity curve and hence an approximate inflection point at $3 \mathrm{wt} \%$ of GNP is assumed as the percolation threshold. The inset shows log-log plot of the data for $\phi>\phi_{\mathrm{c}}$ using a linear fit, where critical exponent $t$ is observed to be 1.5. While value of $t$ varies from 1.6 to 2.0 theoretically, experimental values between 1.3 to 4.0 was reported for three dimensional percolative network of conductive fillers in an insulating matrix [34].

\subsection{Piezoresistive behaviour}

Figure 5 shows the percentage change in resistance $(\Delta R / R)$ of GNP/PMMA films against tensile strain up to a maximum of 1000 micro strains with varying GNP content. It is assumed that the films experience the same strain as that of the beam. Here the change in resistance increases linearly with strain for all films. The slope of the curve keep on increasing up to $3 \mathrm{wt} \%$ and then shows a downward trend with further increase in GNP. This observation is contrary to the earlier study [23], where a monotonous increase in the change resistance is reported with decrease in number density of the GNP. Maximum slope was observed for $3 \mathrm{wt} \%$ GNP sample which showed a significant difference compared to 2.5 and $3.5 \mathrm{GNP}$ wt $\%$ samples. This difference in slope is in turn very large compared to that of the other samples.

The piezoresistive effect of the GNP/PMMA films can be attributed mainly to three factors such as inherent piezoresistivity of the GNP, variation in their conducting network and change in GNP inter-particle distance and tunneling resistance caused by applied strain. At lower concentrations, say below $3 \mathrm{wt} \%$ the distance between the neighboring platelets is relatively large. When this film is strained, the graphene platelets get stretched leading to change in carboncarbon bond lengths resulting in change in resistance owing to increased band gap. This change in resistance does not get transferred to the probes placed a few millimeters apart because of the insulating nature of the matrix rich regions and hence the net change in resistance is observed to be smaller. However, the order of change in resistance is significantly larger compared to a conventional strain gauge and a CNT paint sensor, as shown in the inset. Lee et al. [21] shown that the piezoresistive effect of pristine graphene is limited by the lattice distortion. However, the order of change in resistance is significantly larger compared to pristine graphene. A possible reason could be that the change in the inter particle distance of GNPs coupled with lattice distortion has resulted a higher change in resistance compared to the pristine graphene.

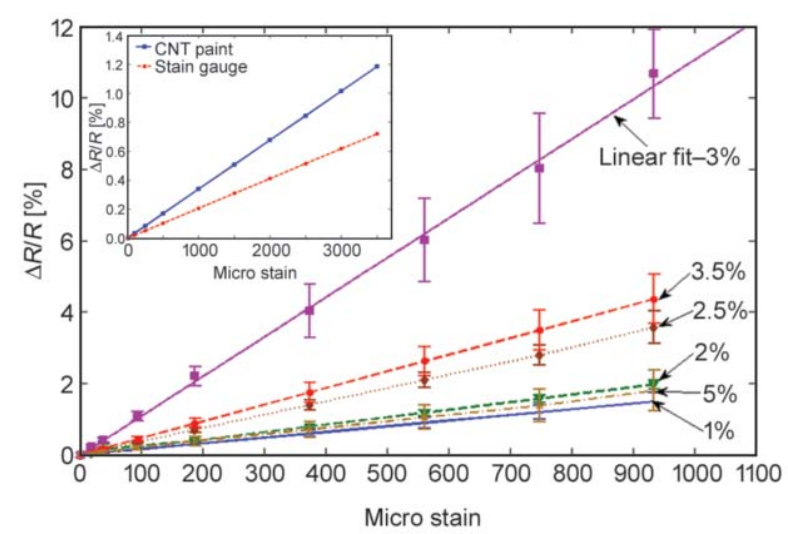

Figure 5. Change in resistance of GNP/PMMA nanocomposite films of different weight percent GNP under tensile strain (Inset shows the response of a CNT paint sensor which is having $8 \mathrm{wt} \% \mathrm{CNT}$ and a standard strain gauge) 
As the GNP content is increased the distance between the neighboring GNP reduces and additional conductive paths are formed. At a critical weight percent i.e. at the percolation threshold of $3 \mathrm{wt} \%$ of GNP, the distance between the neighboring platelets reaches an optimum value that is required for electrical conductivity by tunneling effect. At this point if the film is strained, all the three mechanisms discussed earlier simultaneously comes into effect resulting in a drastic change in resistance with strain. Hempel et al. [23] observed no shift in the Raman G band with strain indicating absence of any deformation of the graphene platelets. Here, in the absence of a polymer matrix to transfer the applied strain to the graphene flakes, the change in resistance has been explained based on the separation between the neighboring flakes caused by their slippage.

When the GNP content is increased beyond the percolation threshold the GNP platelets show a tendency to stack so as to accommodate the excess platelets. Such a composite film when subjected to strain, GNPs move apart creating gaps between the platelets. Subsequently many platelets in the stacked structure slide over into these gaps leading to formation of new conducting pathways. Thus the net change in resistance due to strain is observed to be smaller. This explains the drastic drop in the slope of the piezoresistance curve above the percolation threshold. This strong relation between percolation threshold and piezoresistive strain sensitivity observed in this study is very important in deciding the optimum concentration of GNP in the composites required for developing a strain gauge of high sensitivity for SHM applications.

\subsection{Gauge factor}

The sensitivity of the sensor is measured as gauge factor (GF) which is the ratio of the change in resistance to the change in corresponding strain as shown by Equation (2):

$G F=\frac{R_{\mathrm{f}}-R_{0}}{R_{0} \varepsilon}$

where $R_{0}$ is the unstrained resistance of the sensor, $R_{\mathrm{f}}$ is the final resistance after application of strain $\varepsilon$. Figure 6 shows the GF of films plotted as a function of $\phi / \phi_{c}$, ratio of GNP wt $\%$ to the percolation threshold. For $\phi<\phi_{\mathrm{c}}$, the GF shows an increasing trend with GNP concentration. When $\phi / \phi_{\mathrm{c}}$ is equals to 1 i.e. at the percolation threshold of $3 \mathrm{wt} \%$ of GNP loading, a

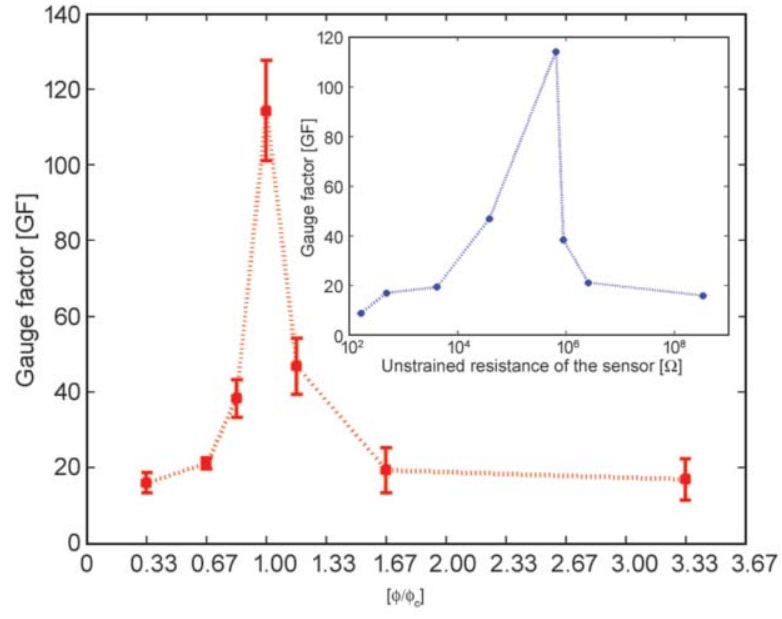

Figure 6. Variation in gauge factor (GF) of nanocomposite films as a function of $\phi / \phi_{\mathrm{c}}$, maximum GF was obtained at $\phi / \phi_{c}=1$, i.e. at the percolation threshold of $3 \mathrm{wt} \%$ of GNP. (Inset shows the variation of GF as a function of unstrained resistance of the sensor.)

maximum sensitivity is obtained with a GF of $114 \pm 13$. On further loading of GNP beyond percolation threshold i.e. $\phi>\phi_{\mathrm{c}}$, the GF is drastically reduced.

This interesting behaviour is attributed to the fact that in the percolation region, tunneling type of effects dominates and up on straining these contacts break and a drastic change in electrical conductivity with strain can be observed. A small change in GNP weight fraction either side of the threshold has shown a significant variation in the GF as shown in the figure. For $\phi<\phi_{c}$, the film was dominated by matrix rich regions and for $\phi>\phi_{c}$, several alternative conductive paths are available for the current to flow and hence a smaller change in resistance with strain is observed in both cases. Details of GFs obtained with wt $\%$ of GNP and unstrained resistance of the sensor are shown

Table 1. Summary of electrical conductivity, unstrained resistance and gauge factors of GNP/PMMA nanocomposite films, standard strain gauge and CNT paint sensor

\begin{tabular}{|c|c|c|c|}
\hline $\begin{array}{c}\text { Weight percent of } \\
\text { GNP in } \\
\text { PMMA/sensor } \\
\text { type }\end{array}$ & $\begin{array}{c}\text { Electrical } \\
\text { conductivity } \\
{[\mathbf{S} / \mathbf{m}]}\end{array}$ & $\begin{array}{c}\text { Sensor } \\
\text { unstrained } \\
\text { resistance } \\
{[\mathbf{\Omega}]}\end{array}$ & $\begin{array}{c}\text { Gauge } \\
\text { factor } \\
{[\mathbf{G F}]}\end{array}$ \\
\hline 1.0 & $6 \cdot 10^{-4}$ & $3.4 \cdot 10^{8}$ & $16 \pm 3.0$ \\
\hline 2.0 & 0.004 & $2.6 \cdot 10^{6}$ & $21 \pm 1.5$ \\
\hline 2.5 & 0.022 & $0.9 \cdot 10^{6}$ & $38 \pm 5.0$ \\
\hline 3.0 & 0.029 & $6.6 \cdot 10^{5}$ & $114 \pm 13$ \\
\hline 3.5 & 0.051 & $3.9 \cdot 10^{4}$ & $47 \pm 7.0$ \\
\hline 5.0 & 9.400 & $4.2 \cdot 10^{3}$ & $19 \pm 6.0$ \\
\hline 10.0 & 42.800 & 488 & $17 \pm 6.0$ \\
\hline 20.0 & 126.000 & 166 & $9 \pm 2.0$ \\
\hline Strain gauge & - & 351 & 2.0 \\
\hline CNT Paint & 115.000 & 131 & 3.4 \\
\hline
\end{tabular}


in Table 1.While the GFs of the standard strain gauge and CNT paint sensors are 2 and 3.4 respectively, a $1 \mathrm{wt} \%$ GNP/PMMA film showed significantly high sensitivity to strain with a GF of 16 . The large noise in the data can be attributed to measurement error. Hempel et al. [23] and Zhao et al. [26] reported an increasing trend for GF as a function of unstrained resistance of the sensor and observed that high resistivity samples exhibit a larger sensitivity to strain [23]. The present study shows (inset of Figure 6) a similar increasing trend with GF but only up to a critical resistance corresponding to the percolation threshold. However, the GF rapidly decreases on further increase in unstrained resistance.

\section{Conclusions}

This study on GNP/PMMA nanocomposite films shows a non-monotonic piezoresistive behaviour and GF with the GNP concentration. Electrical conductivity of the PMMA films followed a power law dependency with a percolation threshold at $3 \mathrm{wt} \%$ of GNP. A significant variation in the piezoresistive strain sensitivity was observed near the percolation region. Contrary to the earlier reports, we observed that the GF is increased with unstrained resistance of the nanocomposite films up to a critical value corresponding to the percolation threshold and then decreased drastically. While a maximum GF of $114 \pm 13$ was obtained for the GNP/PMMA films, commercially obtained CNT paint showed a GF of 3.4. This study substantiates the critical relation between the percolation threshold and piezoresistive behaviour and is useful for the design and development of graphene/ polymer nanocomposite strain sensors and smart structures.

\section{References}

[1] Rawal S., Ravine J., Czerw R.: Graphene nanoplatelet membrane for aerospace applications. Nanotech, 1, 411-414 (2011).

[2] Bao Q., Zhang H., Yang J-X., Wang S., Tang D. Y., Jose R., Ramakrishna S., Lim C. T., Loh K. P.: Graphenepolymer nanofiber membrane for ultrafast photonics. Advanced Functional Materials, 20, 782-791 (2010). https://doi.org/10.1002/adfm.200901658

[3] Batrakov K., Kuzhir P., Maksimenko S., Paddubakya A., Voronovich S., Lambin P., Kaplas T., Svirko Y.: Flexible transparent graphene/polymer multilayers for efficient electromagnetic field absorption. Scientific Reports, 4, 7191/1-7191/5 (2014).

https://doi.org/10.1038/srep07191
[4] Watanabe A. O., Jeong S., Kim S., Kim Y., Min J., Wong D., Pulugurtha M. R., Mullapudi R., Kim J., Tummala R. R.: Highly-effective integrated EMI shields with graphene and nanomagnetic multilayered composites. in 'IEEE 66th Electronics Components and Technology Conference, Las Vegas, USA' 206-210 (2016). https://doi.org/10.1109/ECTC.2016.294

[5] Hill E. W., Vijayaraghavan A., Novoselov K.: Graphene sensors. IEEE Sensors Journal, 11, 3161-3170 (2011). https://doi.org/10.1109/JSEN.2011.2167608

[6] Kim K., Lee Z., Regan W., Kisielowski C., Crommie M. F., Zettl A.: Grain boundary mapping in polycrystalline graphene. ACS Nano, 5, 2142-2146 (2011). https://doi.org/10.1021/nn1033423

[7] Lee C., Wei X. D., Kysar J. W., Hone J.: Measurement of the elastic properties and intrinsic strength of monolayer graphene. Science, 321, 385-388 (2008). https://doi.org/10.1126/science.1157996

[8] Zhu Y., Murali S., Cai W., Li X., Suk J. W., Potts J. R., Ruoff R. S.: Graphene-based materials: Graphene and graphene oxide: Synthesis, properties, and applications. Advanced Materials, 22, 3906-3924 (2010). https://doi.org/10.1002/adma.201090113

[9] Compton O. C., Nguyen S. B. T.: Graphene oxide, highly reduced graphene oxide, and graphene: Versatile building blocks for carbon-based materials. Small, 6, 711-723 (2010). https://doi.org/10.1002/smll.200901934

[10] Wang Y., Yang R., Shi Z., Zhang L., Shi D., Wang E., Zhang G.: Super-elastic graphene ripples for flexible strain sensors. ACS nano, 5, 3645-3650 (2011). https://doi.org/10.1021/nn103523t

[11] Du X., Skachko I., Barker A., Andrei E. Y.: Approaching ballistic transport in suspended graphene. Nature Nanotechnology, 3, 491-495 (2008). https://doi.org/10.1038/nnano.2008.199

[12] Sadasivuni K. K., Ponnamma D., Kim J., Thomas S.: Graphene-based polymer nanocomposites in electronics. Springer, Heidelberg (2015). https://doi.org/10.1007/978-3-319-13875-6

[13] Higginbotham A. L., Lomeda J. R., Morgan A. B., Tour J. M.: Graphite oxide flame-retardant polymer nanocomposites. Applied Materials and Interfaces, 1, 22562261 (2009). https://doi.org/10.1021/am900419m

[14] Chen D., Zhu H., Liu T.: In situ thermal preparation of polyimide nanocomposite films containing functionalized graphene sheets. ACS Applied Materials and Interfaces, 2, 3702-3708 (2010). https://doi.org/10.1021/am1008437

[15] Ramanathan T., Abdala A. A., Stankovich S., Dikin D. A., Herrera-Alonso M., Piner R. D., Adamson D. H., Schniepp H. C., Chen X., Ruoff R. S., Nguyen S. T., Aksay I. A., Prud'Homme R. K., Brinson L. C.: Functionalized graphene sheets for polymer nanocomposites. Nature Nanotechnology, 3, 327-331 (2008). https://doi.org/10.1038/nnano.2008.96 
[16] Tjong S. C.: Graphene and its derivatives: Novel materials for forming functional polymer nanocomposites. Express Polymer Letters, 10, 437 (2012). https://doi.org/10.3144/expresspolymlett.2012.46

[17] Wang M., Yan C., Ma L.: Graphene nanocomposites. in 'Composites and their properties' (ed.: Hu N.) Intech, Rijeka 17-36 (2012). https://doi.org/10.5772/50840

[18] Liu K., Chen S., Luo Y., Jia D., Gao H., Hu G., Liu L.: Edge-functionalized graphene as reinforcement of epoxybased conductive composite for electrical interconnects. Composites Science and Technology, 88, 84-91 (2013). https://doi.org/10.1016/j.compscitech.2013.08.032

[19] Wajid A. S., Tanvir Ahmed H. S., Das S., Irin F., Jankowski A. F., Green M. J.: High-performance pristine graphene/epoxy composites with enhanced mechanical and electrical properties. Macromolecules Materials and Engineering, 298, 339-347 (2013). https://doi.org/10.1002/mame.201200043

[20] Zhao J., Zhang G-Y., Shi D-X.: Review of graphenebased strain sensors. Chinese Physics B, 22, 5770157709 (2013).

https://doi.org/10.1088/1674-1056/22/5/057701

[21] Lee Y., Bae S., Jang H., Jang S., Zhu S-E., Sim S. H., Song Y. I., Hong B. H., Ahn J-H.: Wafer-scale synthesis and transfer of graphene films. Nano Letters, 10, 490493 (2010).

https://doi.org/10.1021/n1903272n

[22] Fu X-W., Liao Z-M., Zhou J-X., Zhou Y-B., Wu H-C., Zhang R., Jing G., Xu J., Wu X., Guo W., Yu D.: Strain dependent resistance in chemical vapor deposition grown graphene. Applied Physics Letters, 99, 213107/1213107/3 (2011).

https://doi.org/10.1063/1.3663969

[23] Hempel M., Nezich D., Kong J., Hofmann A.: A novel class of strain gauges based on layered percolative films of 2D materials. Nano Letters, 12, 5714-5718 (2012). https://doi.org/10.1021/nl302959a

[24] Eswaraiah V., Balasubramaniam K., Ramaprabhu S.: One-pot synthesis of conducting graphene-polymer composites and their strain sensing application. Nanoscale, 4, 1258-1262 (2012).

https://doi.org/10.1039/C2NR11555G

[25] Eswaraiah V., Jyothirmayee Aravind S. S., Balasubramaniam K., Ramaprabhu S.: Graphene-functionalized carbon nanotubes for conducting polymer nanocomposites and their improved strain sensing properties. Macromolecular Chemistry and Physics, 214, 2439-2444 (2013).

https://doi.org/10.1002/macp.201300242
[26] Zhao J., He C. L., Yang R., Shi Z. W., Cheng M., Yang W., Xie G., Wang D., Shi D., Zhang G.: Ultra-sensitive strain sensors based on piezoresistive nanographene films. Applied Physics Letters, 10, 063112/1-063112/5 (2012).

https://doi.org/10.1063/1.4742331

[27] Villar-Rodil S., Paredes J. I., Martínez-Alonso A., Tascón J. M. D.: Preparation of graphene dispersions and graphene-polymer composites in organic media. Journal of Materials Chemistry, 19, 3591-3593 (2009). https://doi.org/10.1039/B904935E

[28] Kim H., Macosko C. W.: Processing-property relationships of polycarbonate/graphene composites. Polymer, 50, 3797-3809 (2009).

https://doi.org/10.1016/j.polymer.2009.05.038

[29] Liang J., Wang Y., Huang Y., Ma Y., Liu Z., Cai J., Zhang C., Gao H., Chen Y.: Electromagnetic interference shielding of graphene/epoxy composites. Carbon, 47, 922-925 (2009). https://doi.org/10.1016/j.carbon.2008.12.038

[30] Pang H., Chen T., Zhang G., Zeng B., Li Z-M.: An electrically conducting polymer/graphene composite with a very low percolation threshold. Materials Letters, 64, 2226-2229 (2010).

https://doi.org/10.1016/j.matlet.2010.07.001

[31] Galpaya D., Wang M., Liu M., Motta N., Waclawik E., Yan C.: Recent advances in fabrication and characterization of graphene-polymer nanocomposites. Graphene, 1, 30-46 (2012). https://doi.org/10.4236/graphene.2012.12005

[32] Kuilla T., Bhadra S., Yao D., Kim N. H., Bose S., Lee J. H.: Recent advances in graphene based polymer composites. Progress in Polymer Science, 35, 1350-1375 (2010). https://doi.org/10.1016/j.progpolymsci.2010.07.005

[33] Pham V. H., Dang T. T., Hur S. H., Kim E. J., Chung J. S.: Highly conductive poly(methyl methacrylate) (PMMA)-reduced graphene oxide composite prepared by self-assembly of PMMA latex and graphene oxide through electrostatic interaction. ACS Applied Materials and Interfaces, 4, 2630-2636 (2012). https://doi.org/10.1021/am300297j

[34] da Silva Leite Coelho P. H., Marchesin M. S., Morales A. R., Bartoli J. R.: Electrical percolation, morphological and dispersion properties of MWCNT/PMMA nanocomposites. Materials Research, 17, 127-132 (2014). https://doi.org/10.1590/S1516-14392014005000059 